\title{
Confined granular flows on a heap: from simulations to experiments
}

\author{
Patrick RICHARD ${ }^{1, \star}$, Alexandre VALANCE ${ }^{2, \star \star}$, Renaud DELANNAY ${ }^{2, \star \star \star}$, and Philippe BOLTENHAGEN ${ }^{2, \star \star \star \star}$ \\ ${ }^{1}$ LUNAM Université, IFSTTAR, MAST, GPEM, F-44340 Bouguenais, France \\ ${ }^{2}$ Institut de Physique de Rennes, Université Rennes 1, UMR CNRS 6251, F-35042 Rennes, France
}

\begin{abstract}
Surface granular flows are studied by means of discrete element simulations. The investigation of their kinematic properties suggests the existence of two regimes: one close to the jamming, for which the shear rate increases with the angle of the flow, and another one, far from jamming, for which the shear remains constant while the flow rate increases. Although the simulations reproduce experimental results for a given gap between sidewalls, they fail to capture the effect of confinement on the flows' properties (shear rate, flow height...).
\end{abstract}

\section{Introduction}

Significant progress has been made during the last decades in understanding gravity driven granular flows; nevertheless a full understanding of their behavior is far from being attained and their study remains an active field of research [1]. Here, we focus on steady and fully developed surface flows confined between two vertical sidewalls, i.e. systems for which the flowing particles appear to be limited to a surface layer above a "frozen" bulk region. In such systems, there is a critical flow rate above which the flow occurs on a static heap which forms along the base [2]. Its angle, $\theta$, is determined by the flow rate, the internal dissipation of the flow and its friction with sidewalls. Using a balance of momentum for the flowing layer, one can derive an affine scaling law linking $\theta$, the height of the layer, $h$, and the width of the channel, $W$ :

$$
\tan \theta=\mu_{I}+\mu_{W} h / W
$$

where $\mu_{I}$ and $\mu_{W}$ are effective friction coefficients [2]. Flows atop this sidewall-stabilized heap (SSH) differ fundamentally from flows on bumpy bases as they occur over erodible bases. However they still display steady and fully developed (SFD) features. The sidewall effect is not limited to important confinements (i.e. small gaps between sidewalls) since it has been experimentally quantified for flows on top of a static pile in wide channels [3]. We have performed a systematic, large-scale simulation study of SFD confined surface flows of cohesionless granular media in three dimensions. In agreement with the literature, we show that SSH flows can be attained for a wide range of angles. Interestingly at large angles the solidlike, liquidlike and gaslike behaviors coexist. This type of flows

\footnotetext{
^e-mail: patrick.richard@ifsttar.fr

$\star \star$ e-mail: alexandre.valance@univ-rennes1.fr

$\star \star \star$ e-mail: renaud.delannay@univ-rennes1.fr

$\star \star \star \star$ e-mail: philippe.boltenhagen@univ-rennes1.fr
}

is therefore a relevant choice to test theories aiming to describe this coexistence.

The outline of this paper is the following. The next section is devoted to a short description of the numerical methods. Then, in section 3, we will study the relation between the angle of the flow and the flow rate and how it is influenced by confinement and by a modification of the micromechanical parameters (coefficients of restitution and friction). The kinematic properties of the flow will be discussed in section 4. Finally, in section 5, we compare our numerical results with experimental ones. In particular we show that even if we adjust the micromechanical parameters to match the experimental results for a given gap between sidewalls, $W$, our simulations are unable to capture the experimental dependence of the flow properties with $W$. This result questions the classical implementation of contact laws in soft sphere Discrete Element Method (DEM) simulations.

\section{Numerical methods}

To carry out our simulations we use our own implementation [4] of the classical DEM where Newton's equations of motion for a system of "soft" but stiff grains are integrated. It includes friction and rotation. Since the DEM is well known and can be found in many papers (e.g. among many others [5]), we just present here the forces used in this work. For the normal force between two overlapping spheres we use a standard linear spring-dashpot interaction model: $\mathbf{f}_{n}=k_{n} \boldsymbol{\delta}_{\boldsymbol{n}}-\gamma_{n} \mathbf{v}_{n}$, where $\boldsymbol{\delta}_{\boldsymbol{n}}$ is the normal overlap, $k_{n}$ is the spring constant, $\gamma_{n}$ the damping coefficient and $\mathbf{v}_{n}$ the normal relative velocity. Likewise we model the tangential force (which induces rotation for frictional grains) as a linear elastic and linear dissipative force in the tangential direction: $\mathbf{f}_{t}=-k_{t} \boldsymbol{\delta}_{t}-\gamma_{t} \mathbf{v}_{t}$, where $k_{t}$ is the tangential spring constant, $\boldsymbol{\delta}_{\boldsymbol{t}}$ the tangential overlap (obtained 
by integrating surface relative velocities during elastic deformation of the contact), $\gamma_{t}$ the tangential damping and $\mathbf{v}_{t}$ the tangential velocity at the contact point. The magnitude of $\boldsymbol{\delta}_{\boldsymbol{t}}$ is truncated as necessary [6] to satisfy the Coulomb law, $\left|\mathbf{f}_{t}\right| \leq \mu_{g g}\left|\mathbf{f}_{n}\right|$, where $\mu_{g g}$ is the grain-grain friction coefficient. The granular material is an assembly of $N$ dissipative spheres (average diameter $d=2 R$ and average mass $m$ ) submitted to gravity g. A small polydispersity of $\pm 20 \%$ is considered to prevent crystallization. The following values of the parameters are used: $k_{n} / m g=5.6 \times 10^{6}$, $k_{t}=2 k_{n} / 7, \gamma_{t}=0$. The value of $\gamma_{n}$ is adjusted to obtain the desired value of the normal restitution coefficient $e_{n}$ [7]. The simulation chute (see figure 1) consists of a $3 D$ cell which can be inclined relatively to the horizontal by an angle $\theta$. Its size in $x$-direction (main flow direction) is set to $25.3 d$. In $z$ direction (i.e. normal to the free surface of the flow) the size of the cell is large and considered as infinite. Frictional sidewalls are located at positions $y=0$

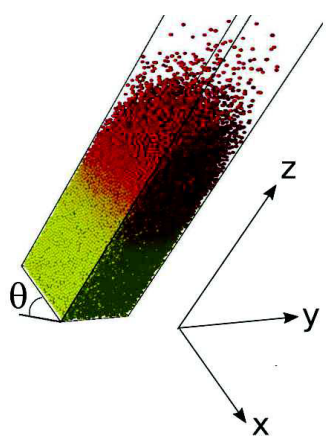

Figure 1. Typical 3D snapshot for chute flow: $W / d=20, N=$ 24,000 . The angle of the flow is $45^{\circ}$, the coefficient of restitution $e_{n}$ is equal to 0.88 , and the friction coefficients $\mu_{g g}$ and $\mu_{g w}$ are set to 0.5 . Flow is directed down the incline.

and $y=W$. They are treated as grains of infinite mass and radius and characterized by the grain-sidewall friction coefficient $\mu_{g w}$. The bottom of the cell is made bumpy by pouring under gravity $\mathbf{g}$ a large number of grains in the cell and by gluing (i.e. setting their mass to infinity) those that are in contact with the plane $z=0$. The initial state is a randomly diluted hexagonal lattice with disorder both on grain positions and velocities. Initially the angle was set to a large angle $\theta \approx 70^{\circ}$ for a significant duration to remove any influence of the initial state and then decreased to the desired value. Our results are given in nondimensional quantities by using $d, \sqrt{d / g}$ and $m$ as units of length, time and mass respectively. The flow domain is partitioned in horizontal strips whose height is equal to the average grain size. The average quantities corresponding to macroscopic field variables are determined for each strip by averaging over time the properties of the grains whose center is located in the considered strip. Although such an averaging method may induce some artefacts when computing properties involving fluctuations (e.g. granular temperature $[8,9])$, it is not the case for the properties reported here (volume fraction and velocity).

\section{Flow rate}

Our initial state being very energetic, the mass of particles flowing out of the cell of the simulation per unit time decreases with time towards a constant value corresponding to a SFD flow which establishes throughout the simulation cell. Within the control parameters investigated so far, we obtain either a SFD flow or a completely jammed state, i.e., no flow. For each cell width $W$ we can therefore define an angle $\theta_{1}$ below which the flow jams. This angle decreases with increasing $W$ and reaches $\theta_{1}^{\infty}$ the "starting angle" classically obtained in case of laterally unbounded flows. Note also that, in vicinity of $\theta_{1}$, the temporal evolution of the flow rate may be intermittent in agreement with experiments [10]. This intermittency induces a significant uncertainty on the determination of $\theta_{1}$. Indeed, an intermittent behavior may be observed for a long period of time before the system comes to rest. It is also important to emphasize here that the presence of flat frictional walls allows the existence of steady flows at much higher angle than $\theta_{2}^{\infty}$, angle defined as the limit angle above which unconfined flows are accelerated [11]. Besides, even at very steep angles, the flow reaches a steady state. It seems that there is no upper limit angle for the existence of steady states for confined flows. These results point out the crucial importance of sidewalls on the behavior of granular flows. Additionally, as already been reported in the literature $(e . g .[12,13])$, the angle $\theta_{1}$ depends on the lateral width $W$ : the wider the gap is the smaller is $\theta_{1}$ [14]. We now focus on the flow rate $Q$ and
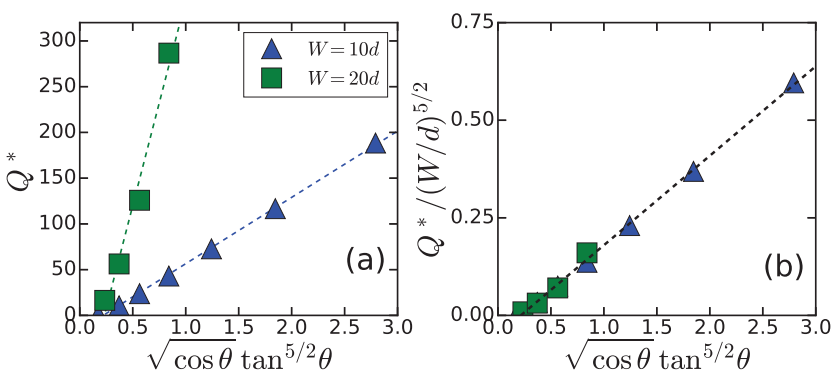

Figure 2. (a) Flow rate per unit width $Q^{\star}$ versus $\sqrt{\cos \theta} \tan ^{5 / 2} \theta$ for different widths $W$. (b) Rescaled flow rate versus $\sqrt{\cos \theta} \tan ^{5 / 2} \theta$

more precisely on the flow rate per unit width, $Q^{\star}=Q / W$ which is an increasing function of the gap width $W$ (figure 2(a)), as a consequence of the declining influence of the wall friction [2,3]. In agreement with [3] we found that $Q^{*}$ scales with $W^{5 / 2}$ (figure 2(b)). Our results are also in reasonable agreement with the analysis reported in [15] where the authors predict a dependance of the flow rate with $\sqrt{\cos \theta} h^{5 / 2}$ i.e. in our case $\sqrt{\cos \theta} \tan ^{5 / 2} \theta$ (see equation 1). We investigated the influence of the microscopic parameters of particle/particle interactions. We varied three parameters (the particle/particle restitution coefficient $e$, the grain/grain Coulomb friction $\mu_{g g}$ and the 
grain/wall friction $\mu_{g w}$ ) and analyzed their respective influence on the flow rate. It turns out that the flow rate is weakly sensitive to the restitution coefficient $e$, except when the elastic limit is approached (i.e., $0.9<e<1$ ). In contrast, the friction coefficients $\mu_{g g}$ and $\mu_{g w}$ have a much more significant impact on the flow rate. For a given $\mu_{g g}$ coefficient, the flow rate exhibits a strong monotonic decrease with increasing grain-wall friction. When $\mu_{g w}$ is increased from 0.1 to 0.3 , the flow rate is decreased by one order of magnitude. Identically, the flow rate decreases with increasing grain-grain friction but at a smaller rate and seems to reach an asymptotic lower limit when $\mu_{g g}$ exceeds $\mu_{g w}$.

\section{Kinematic properties}

In agreement to what has been reported in [4] the vertical profile of the volume fraction (not shown) is found to collapse on a single master curve

$$
v(z)=\frac{v_{0}}{2}\left[1+\tanh \left(z / l_{v}\right)\right],
$$

with origin such that $v=v_{0} / 2$ at $y=0$. In the static pile where $y \gg l_{v}, v \rightarrow v_{0}=0.6$. The characteristic length of the variation of $v, l_{v}$, scales with $W$ and increases with inclination, $l_{v} / W \simeq \eta \times\left[\tan \theta-\tan \theta_{0}\right]$, where $\theta_{0} \approx 17^{\circ}$ and $\eta \approx 1.2$, a relation that we established for $W / d=10$ and 20 (figure 3 ). Note that such a relation has been successfully tested for other confinements $(W / d=5$ and $W / d=30$ ) [14]. Of course, the quantities $\theta_{0}$ and $\eta$ change with $\mu_{g g}$ and $\mu_{g w}$. Two remarks deserve to be mentioned here. First, the variation of the characteristic $l_{v}$ with $\tan \theta$ deviates from an affine law when approaching the jamming transition. Second, the characteristic length $l_{v}$ can be used to define unambiguously the height of the of the flowing layer : $h=2 l_{v}$ which also scales as $W \tan \theta$ as reported in [4].

As expected, the vertical profiles of the velocity are localized at the free surface, with a linear profile at the top followed by an exponential tail. In case of high flow angles a decrease of the velocity in the gaseous layer is also observed (figure 4(a)). Interestingly, these profiles can also be scaled by a characteristic speed which depends on $l_{v}$ : $V_{0} \sim\left(l_{v} / d\right) \sqrt{g W}$ except close to jamming (figure 4(b)). This suggests the existence of two flow regimes, one far from jamming characterized by the length scale $l_{v}$ and a shear rate $\dot{\gamma}=\partial_{y} v_{x}$ which does not depend on $\theta$ and the other close to jamming for which the shear rate increases with $\theta$.

\section{Comparison with experiments}

We carried out experiments with the aim to validate our numerical approach. Glass beads of diameter $d=(500 \pm$ 100) $\mu \mathrm{m}$ are poured between two parallel, vertical glass plates separated by $W$ from a hopper orifice controlling the flow rate, thus fixing $h$ and $\theta$. A Photron APX RS camera of $1024 \times 1024$ resolution for $50-30000 \mathrm{~Hz}$ frame rate and a particle tracking algorithm are used to track rapid grains
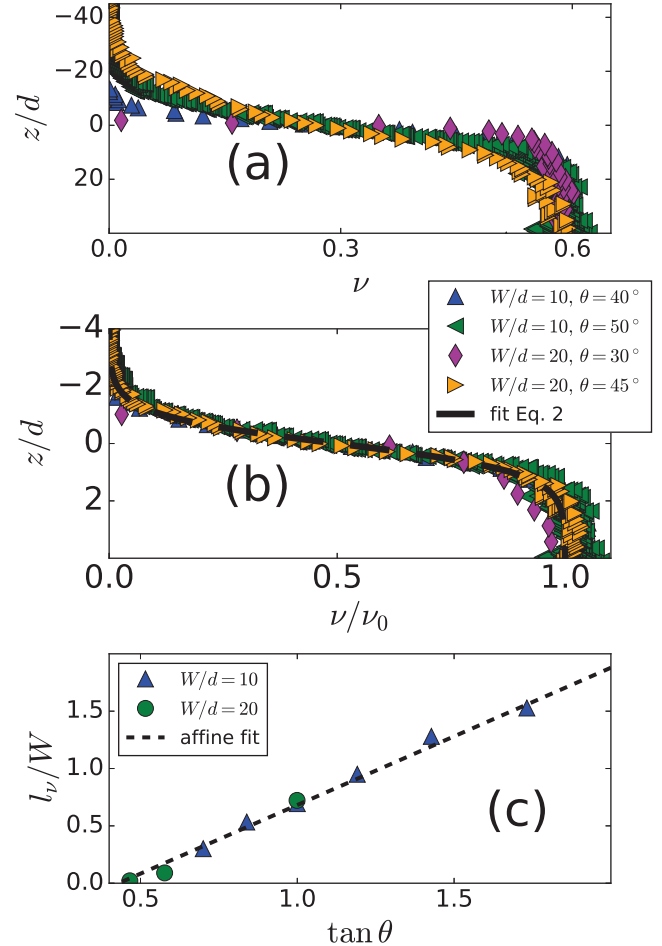

Figure 3. The profiles of volume fraction $v$ at inclinations and gap between sidewalls shown (a) are well fitted by Eq. 2 and can be rescaled into a single curve when plotting $v / v_{0}$ versus $z / l_{v}$ (b), where $l_{v}$ is the characteristic length of the variation of $v$, which scales with $W$ and is affine with $\tan \theta$ (c).
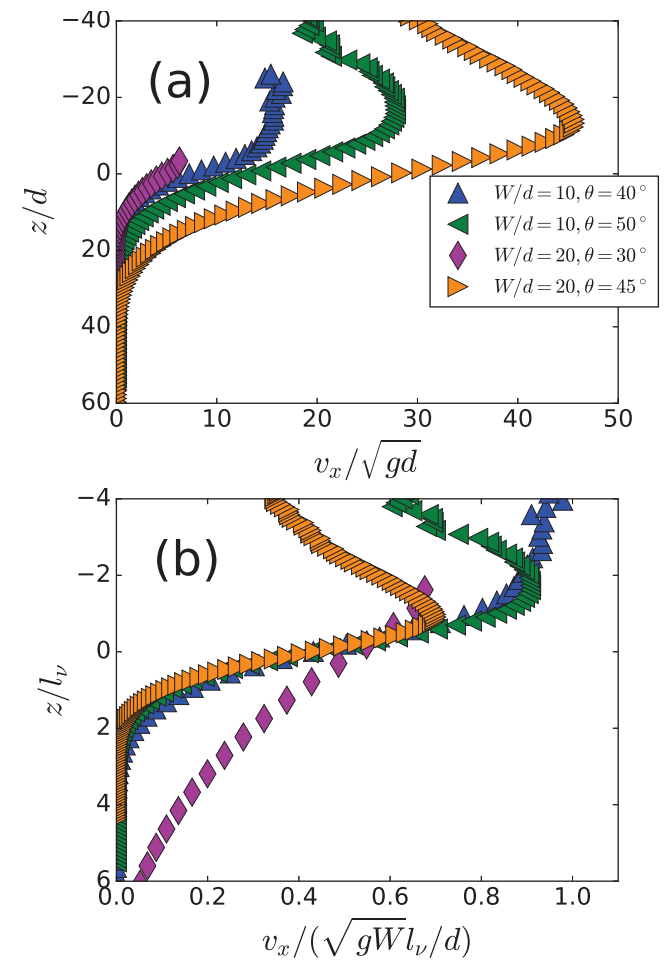

Figure 4. Vertical profiles of the horizontal particle velocity for various inclination angles $\theta$ and widths $W$. 
at the sidewalls. We first adjust the value of $\mu_{g g}$ and $\mu_{g w}$ in our simulations to fit the experimental dependance of the evolution of the flow rate with the angle of the flow $\theta$ (the effect of $e$ is weak). For $W=10 d$, we obtain a good agreement between experiments and numerical simulations by setting the friction coefficients to $\mu_{g g}=0.2$ and $\mu_{g w}=0.48$ (see figure 5(a)). Interestingly, the same values also give
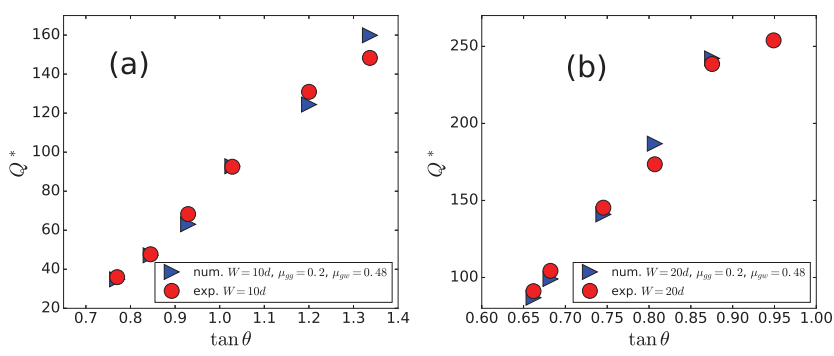

Figure 5. The simulations with $\mu_{g g}=0.2$ and $\mu_{g w}=0.48$ are capable to reproduce the experimental evolution of the flow rate per unit width $Q^{*}$ with the angle of the flow $\theta$ both for $W=10 d$ and $W=20 d$.
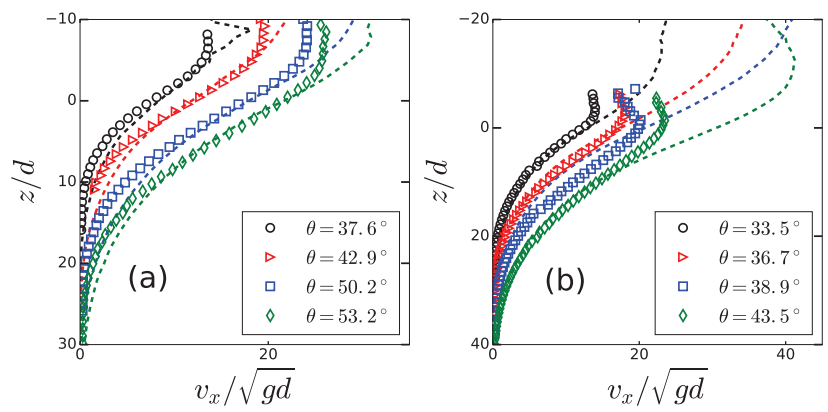

Figure 6. Vertical profiles of the horizontal particle velocity for various inclination angles $\theta$ and for $W=10 d$ and $W=20 d$.

a good fit of the flow rate for $W=20 d$ (figure 5(b)). However, we have to keep in mind that the flow rate is a macroscopic quantity. Consequently we also have to verify that the same friction coefficients allow to reproduce microscopic quantities like velocity profiles. Figure 6(a) shows that the numerical velocity profiles obtained for $W=10 \mathrm{~d}$ matches reasonably the experimental one. The height of the numerical seems to a be a little bit higher than the experimental one but it might be a consequence of the very low volume fraction at the top of the flow which leads to an underestimation of the height in experiments. On the contrary, for $W=20 d$ (figure 6(b)) the numerical flows have a much greater height than the experimental ones, which cannot be explained by the low density region at the top of the flow. Consequently, this result questions the relevancy of such numerical methods to model quantitatively granular flows: our simulations are capable to reproduce the experimental results for a given gap between sidewalls, $W$, -at least qualitatively- but not the experimental dependence of the flow properties with $W$.

\section{Conclusion}

Our study showed that although our simulations reproduce reasonably experiments for a given confinement, the experimental dependence of the flow properties with $W$ is not captured by our simulations. This result questions the way that contact laws are classically implemented in soft sphere DEM simulations. As noticed previously in several works (e.g. [16]) the dissipation of multiparticle contacts is probably much too low. Thus, our work highlights the importance of dissipative force modeling in DEM.

\section{Acknowledgements}

We thank the Centre de Calcul Intensif des Pays de la Loire (CCIPL) for the use of their facilities (project MTEEGD).

\section{References}

[1] R. Delannay, M. Louge, P. Richard, N. Taberlet, A. Valance, Nature Materials 6, 99 (2007)

[2] N. Taberlet, P. Richard, A. Valance, W. Losert, J.M. Pasini, J.T. Jenkins, R. Delannay, Phys. Rev. Lett. 91, 264301 (2003)

[3] P. Jop, Y. Forterre, O. Pouliquen, Journal of Fluid Mechanics 541, 167 (2005)

[4] P. Richard, A. Valance, J.F. Métayer, P. Sanchez, J. Crassous, M. Louge, R. Delannay, Physical Review Letters 101, 248002 ( 4) (2008)

[5] L.E. Silbert, D. Ertaş, G.S. Grest, T.C. Halsey, D. Levine, S.J. Plimpton, Phys. Rev. E 64, 051302 (2001)

[6] L. Brendel, S. Dippel, Lasting Contacts In Molecular Dynamics Simulations, in Physics of Dry Granular Media (Kluwer Academic Publishers, 1998), pp. 313-318

[7] S. Luding, European Journal of Environmental and Civil Engineering - EJECE 12 12, 785 (2008)

[8] H. Xu, A.P. Reeves, M.Y. Louge, Review of Scientific Instruments 75, 811 (2004)

[9] R. Artoni, P. Richard, Phys. Rev. E 91, 032202 (2015)

[10] R. Fischer, P. Gondret, M. Rabaud, Phys. Rev. Lett. 103, 128002 (2009)

[11] GDR-MiDi, Eur. Phys. J. E 14, 341 (2004)

[12] C.h. Liu, H.M. Jaeger, S.R. Nagel, Phys. Rev. A 43, 7091 (1991)

[13] S. Courrech du Pont, P. Gondret, B. Perrin, M. Rabaud, EPL (Europhysics Letters) 61, 492 (2003)

[14] P. Richard, A. Valance, R. Delannay, P. Botltenhagen, in preparation (2016)

[15] J.L. Baker, T. Barker, J.M.N.T. Gray, Journal of Fluid Mechanics 787, 367 (2016)

[16] L. Pournin, T.M. Liebling, A. Mocellin, Phys. Rev. E 65, $011302(2001)$ 